\title{
Autonomous Extrinsic Calibration of a Depth Sensing Camera on Mobile Robots
}

\author{
Farhoud Malekghasemi ${ }^{1}$, Georg Halmetschlager-Funek ${ }^{1}$ and Markus Vincze ${ }^{1}$
}

\begin{abstract}
This work presents a fast and autonomous system to find the rigid transformation between the RGB-D camera and a local reference frame on a mobile robot. The major advantages of the method over the conventional methods of calibration is that there is no need for a special setup or any known object in the scene and its speed. This is achieved by taking advantage of robot's motion combined with camera tracking method. We show that two circular motion and one plane detection are sufficient to autonomously calibrate the robot in the different environments with some minimal texture. The presented method is evaluated with both, computer simulation and in real-life scenarios.
\end{abstract}

\section{INTRODUCTION}

Depth sensing cameras like Microsoft's Kinect, also known as RGB-D cameras, provide robots with threedimensional information (3D) of its environment by using structured infrared light (cf. Fig. 1). Therefore, they have become very popular especially in the branch of mobile robotics because the depth perception is necessary for a successful obstacle avoidance, SLAM, object recognition, segmentation, 3D reconstruction and camera tracking [1][4]. 3D camera collects information from its own perspective (in camera coordinates), which is then transformed to a global coordinate system in order to relate the other parts of the robot to achieve a required task. The transformation is only possible when the relationship between the camera and other parts of the robot are known, therefore most methods require prior knowledge of accurate measurement. The parameters which are used to describe this relationship are called extrinsic parameters of a camera.

In practice, extrinsic parameters of a camera are not always constant and could vary during the time in multiple cases such as wear and tear in robot parts, collision accidents, changing camera's mounting place on robot's body by the user to adopt different environments. All of these displacements violate the prior assumption of known transformations. Thus, recalibration of the camera is necessary. But the process of recalibration is challenging and time-consuming. The state-of-the-art methods of extrinsic calibration are to measure distances directly or using reference objects on the scene with precalibrated position and orientation [5] which are respectively, not accurate and needs a long procedure. These methods are not easily repeatable without an expert

\footnotetext{
${ }^{1}$ All authors are with the V4RLab, ACIN, Faculty of Electrical Engineering, TU Wien, 1040 Vienna, Austria ffarhoud.malekghasemi, georg.halmetschlager, markus.vincze\}@tuwien.ac.at

This work is supported by the European Commission through the Horizon 2020 Programme (H2020-ICT-2014-1, Grant agreement no: 645376).
}

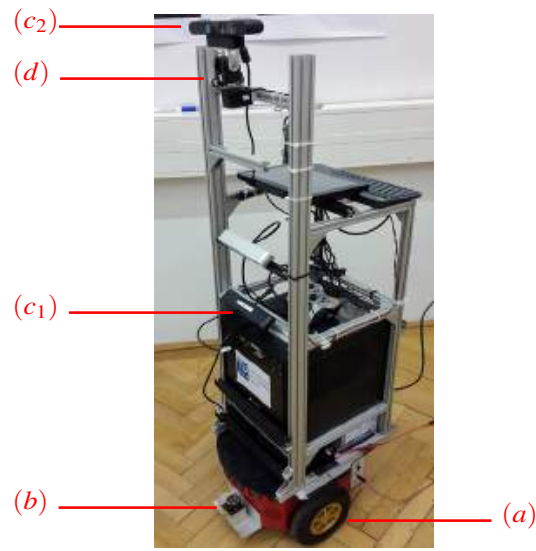

Fig. 1. V4core is a mobile robot system for research and development made by Vision4Robotics group in Vienna University of Technology based on a Pioneer P3-DX platform from MobileRobots company. It is equipped with $(a)$ two differential-drive wheels, $(b)$ scanning laser in front, $\left(c_{1,2}\right)$ Two Asus Xtion RGBD cameras which are mounted at different heights and $(d)$ a pan-tilt unit for top camera.

in the loop, e.g., for filling the images into the the system with showing a calibration template to the cameras.

To overcome these problems we contribute a fast and autonomous method to find the rigid transformation between the RGB-D camera and a reference frame on a mobile robot by taking advantage of its motion combined with visual motions estimation of the camera. We assume the robot's working area is a flat floor with some minimal texture, on which it can freely move around and is observed by the camera.

\section{RELATED WORK}

Most of related works focus on the calibration of intrinsic camera parameter. There exist several methods that use the motion of a camera to calibrate the intrinsic parameters, such as: [6]-[8].

Calibrations without using any specialized reference objects or patterns have also been studied in [9]-[12]. Carrera et al. [10] calibrated a fixed multi RGB camera rig by detecting invariant SURF feature correspondences across different views. In Miller et al. [11] work, the extrinsic parameters were estimated for calibrating the relative pose and time offsets of a pair of depth sensors based on point correspondences established from the unstructured motion of 
objects in the scene. Pathirana et al. [12] proposed a method to calibrate multiple cameras based on users joint positions.

Furthermore, similar techniques are proposed for calibrating $2 \mathrm{D}$ and $3 \mathrm{D}$ LIDAR sensors mounted on a moving platform in [13]-[15].

\section{METHOD}

The proposed method estimates the camera pose in $3 \mathrm{D}$ space by driving the robot in predetermined paths which provide us the required camera trajectories for calculations. The pose of the camera in 3D space is described by translation and rotation with respect to the robot's base coordinate system. Therefore, there are six parameters (6 DoF) to be determined:

- Three translation distances: X, Y, Z

- Three rotation angles: $\operatorname{Roll}(\phi), \operatorname{Pitch}(\theta), \operatorname{Yaw}(\psi)$

We divide these six parameters in three categories as following:

1) $\mathrm{Z}, \phi, \theta$

2) $X, Y$

3) $\psi$

to design a special step for separately calculating parameters of each category. These steps have been named respectively as ground plane detection, two-rotation drive and straightforward drive. Fig. 2 shows an overview of three main steps and relation between sub steps in the algorithm.

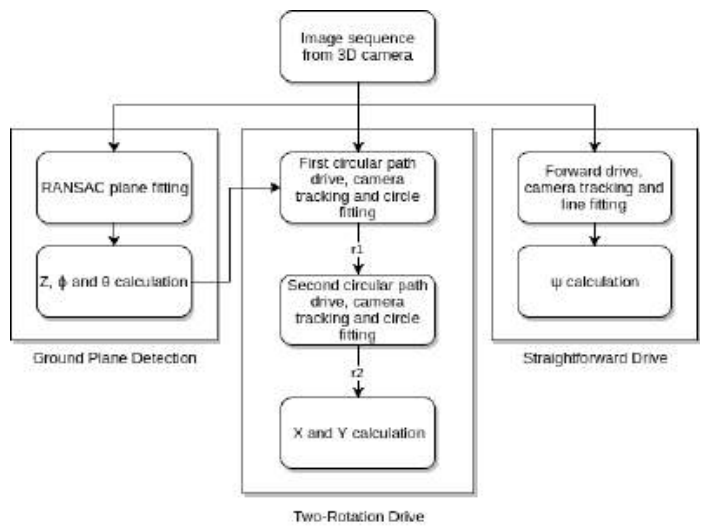

Fig. 2. Overview of different steps in the approach.

\section{A. Camera Tracker}

A camera tracking algorithm provides us the trajectory of the camera in 3D space. This trajectory is determined using visual motion estimation with the suggested method in [16]. The method combines two approaches:

- Feature-based method using a pyramidal implementation of the KLT-tracker [17].

- A keyframe-based refinement step.

It detects FAST-keypoints [18] first to initialize a keyframe and assign them to the corresponding 3D locations. Then it tracks, frame by frame, the keypoints using pyramidal KLT-tracker, which allows tracking large camera motions. Finally, it uses RANSAC to robustly estimate the rigid transformation (the camera pose) from the corresponding depth information of the organized RGB-D frames. Additionally, it applies a keyframe-based refinement step by projecting patches to the current frame to account for the accumulated drift for individual point correspondences and optimizing their locations. The algorithm produces as output a set of keyframes $K=\left\{K^{1}, \ldots, K^{n}\right\}$ and a set of transformations $T=\left\{T^{1}, \ldots, T^{n}\right\}$ for camera pose adjusting the corresponding keyframes to the reference frame which is defined by the first camera frame or by the user.

\section{B. Ground Plane Detection}

We assumed that the robot is working on a flat floor. Detecting this one plane in the camera's field of view is enough to calculate the parameters of the first category. The segmentation algorithm finds all the points within a point cloud that supports a plane model using the random sample consensus (RANSAC) [19] as a robust estimator of choice. A threshold for distance determines how close a point must be to the model in order to be considered as an inlier. Finally the contents of the inlier set, are used to estimate coefficients of plane's equation in 3D space:

$$
n_{x} x+n_{y} y+n_{z} z+d=0
$$

wherein $d$ represents the distance between the plane and the camera, which is equivalent to the distance of the camera from the ground (height of the camera), also known as $\mathrm{Z}$ parameter in this category. The vector $\mathbf{n}^{T}=\left[n_{x}, n_{y}, n_{z}\right]$ represents the normalized normal vector of the plane which is perpendicular to the surface. The formed angles between this normal vector and the camera's coordinate system provide the roll and pitch angles in this category, which can be simply calculated using trigonometry as illustrated in Fig. 3.

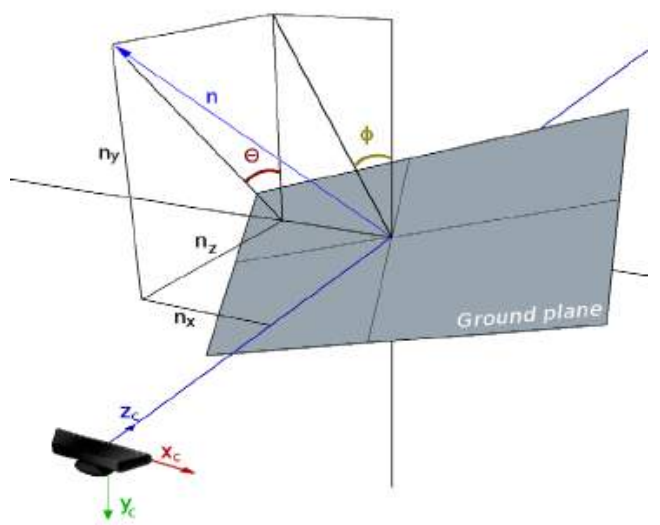

Fig. 3. $\operatorname{Roll}(\phi), \operatorname{Pitch}(\theta)$ with respect to detected ground plane in camera coordinate system. 
The pitch angle of camera $\theta$ is equal to the angle between the normal vector of the ground plane $\mathbf{n}$ and the $x_{c}-y_{c}$ plane of camera coordinate system, so it is calculated with:

$$
\boldsymbol{\theta}=\arctan \left(\frac{n_{z}}{n_{y}}\right)
$$

The roll angle of camera $\phi$ is equal to the angle between the normal vector of the ground plane $\mathbf{n}$ and the $y_{c}-z_{c}$ plane in the camera coordinate system, so it is calculated with:

$$
\phi=\arctan \left(\frac{n_{x}}{n_{y}}\right) .
$$

\section{Two-Rotation Drive}

This method is used to calculate the second category parameters, including $\mathrm{X}$ and $\mathrm{Y}$ distances of the camera in the robot base coordinate system. In case of mobile robots, the base coordinate system is usually chosen to be at the robot's center of mass. In this step, the robot rotates along two circular paths with different radiuses. If the trajectory of the camera is determined during these rotations, then $\mathrm{X}$ and $\mathrm{Y}$ distances can be calculated using simple geometry.

The camera tracker provides these transformations from camera perspective in the base frame of the robot, which we selected as the reference frame. Before any calculation is started the camera trajectory should be transformed to compensate for roll and pitch angels that have been found in the previous section since the camera coordinate and robot base coordinate systems are rotated respectively.

When the robot drives two times with circular path, the camera also has circular movement trajectories with respect to the center of rotation. Radiuses for these two drive can be chosen arbitrary but they should not be equal distances. In order to reduce path execution error and noise production during the drive, rotation radius is selected to be zero for the first drive and half of the distance between two wheels for the second drive. In the first rotation, the robot rotates exactly around itself on a spot and the center of rotation will be equal to the base origin. Then in the second rotation, it will rotate exactly around one of the wheels which keeps this wheel's motor off. Fig. 4 shows a robot from top view with two differential-drive wheels in the base coordinate system and two camera trajectories that would be taken during the first and second circular path drive by the camera mounted on it.

The camera trajectory is a set of points $T$ in $3 \mathrm{D}$ space in camera coordinate system which has rotation with respect to base coordinate system. Therefore before any further more calculation this trajectory should be rotated using roll and pitch angels determined in previous section. The yaw angle does not effect calculation because we are only interested in magnitude of circle's radius and since the camera height is fixed trajectory's data in the $z$ direction is also irrelevant here. $r_{1}$ and $r_{2}$ radiuses can be calculated by applying 2D circle fit algorithm on this set of points.

The algorithm is an implementation of direct least squares fitting a circle to $2 \mathrm{D}$ points in [20]. The goal is to fit a set of points with a circle equation:

$$
(x-a)^{2}+(y-b)^{2}=r^{2}
$$

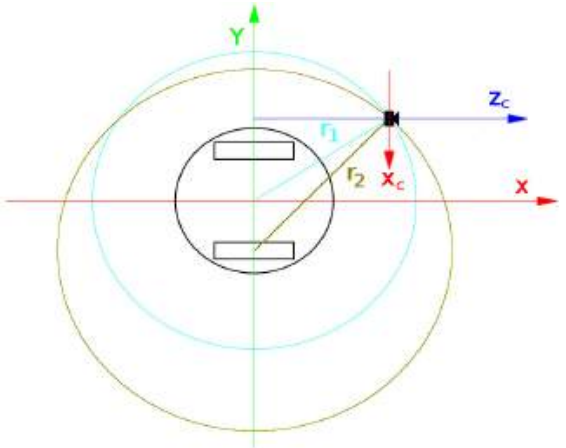

Fig. 4. Top view of a robot with two differential-drive wheels in the base coordinate system and a 3D camera on board for Two-Rotation drive.

where $[a, b]^{T}$ is the circle center and $r$ is the circle radius. The error function to be minimized for $n$ points in the set is:

$$
E=\sum_{i=1}^{n}\left(L_{i}-r\right)^{2}
$$

where $L_{i}=\sqrt{\left(x_{i}-a\right)^{2}+\left(y_{i}-b\right)^{2}}$. Setting to zero of partial derivatives of equation 5 with respect to $a, b$ and $r$ leads to:

$$
\begin{gathered}
r=\frac{1}{m} \sum_{i=1}^{n} L_{i} \\
a=\frac{1}{m} \sum_{i=1}^{n} x_{i}+r \frac{1}{m} \sum_{i=1}^{n} \frac{\partial L_{i}}{\partial a} \\
b=\frac{1}{m} \sum_{i=1}^{n} y_{i}+r \frac{1}{m} \sum_{i=1}^{n} \frac{\partial L_{i}}{\partial b} .
\end{gathered}
$$

These equations can be solved using fixed-point iteration to obtain radius $r$ and center of the circle.

After calculation of $r_{1}$ and $r_{2}$, the two camera trajectories equations in the $x-y$ plane can be written as below:

$$
\begin{aligned}
& x^{2}+\left(y-r_{F}\right)^{2}=r_{1}^{2} \\
& x^{2}+\left(y-r_{S}\right)^{2}=r_{2}^{2}
\end{aligned}
$$

with $r_{F}$ and $r_{S}$ are the first and second drive radiuses. $\mathrm{X}$ and $\mathrm{Y}$ distances are calculated by solving two circles equations for an intersection point as the flowing:

$$
\begin{gathered}
Y=\frac{r_{1}^{2}-r_{2}^{2}+r_{S}^{2}-r_{F}^{2}}{2\left(r_{S}-r_{F}\right)} \\
X=\sqrt{r_{1}^{2}-\left(Y-r_{F}\right)^{2}} .
\end{gathered}
$$

We assume that the camera is looking forward on the robot, therefore the calculated negative value for $\mathrm{X}$ will be discarded. 


\section{Straightforward Drive}

This method is used to calculate the yaw angle of the camera in the last category. For this calculation, the robot starts driving straightforward for a short distance while the camera tracker is providing the camera trajectory. As shown in Fig. 5, considering camera trajectory with respect to the camera coordinate system forms a line in the $z_{c}-x_{c}$ plane. Calculation of the angle between $z_{c}$ axis of the camera and this line yields to the yaw angle. In order to get the slope of the trajectory, a line fit algorithm can be applied to the set of camera trajectory points.

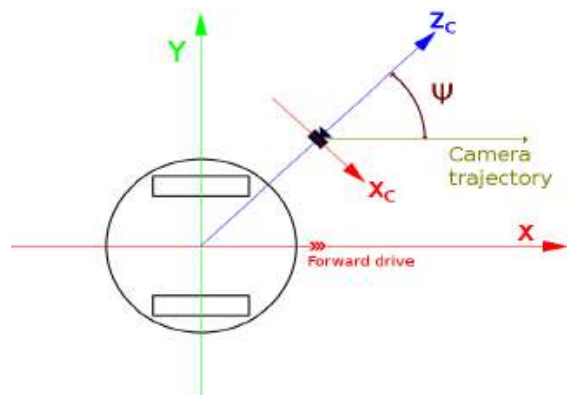

Fig. 5. Top view of a robot with two differential-drive wheels in the base coordinate system and a $3 \mathrm{D}$ camera on board for straightforward drive.

The line fit algorithm is an implementation of linear fitting of $2 \mathrm{D}$ points in [20]. The goal is to fit a set of points with a line equation:

$$
y=A x+B .
$$

The error function to be minimized is the sum of the squared errors between the $y$ values and the line values (only in $y$ direction).

$$
E=\sum_{i=1}^{n}\left[\left(A x_{i}+B\right)-y_{i}\right]^{2}
$$

Setting gradient of equation 14 to zero leads to a system of two linear equations:

$$
\left[\begin{array}{cc}
\sum_{i=1}^{n} x_{i}^{2} & \sum_{i=1}^{n} x_{i} \\
\sum_{i=1}^{n} x_{i} & n
\end{array}\right]\left[\begin{array}{c}
A \\
B
\end{array}\right]\left[\begin{array}{c}
\sum_{i=1}^{n} x_{i} y_{i} \\
\sum_{i=1}^{n} y_{i}
\end{array}\right]
$$

which can be solved to obtain $A$ and $B$.

$$
\begin{gathered}
A=\frac{n \sum_{i=1}^{n} x_{i} y_{i}-\sum_{i=1}^{n} x_{i} \sum_{i=1}^{n} y_{i}}{n \sum_{i=1}^{n} x_{i}^{2}-\sum_{i=1}^{n} x_{i} \sum_{i=1}^{n} x_{i}} \\
B=\frac{\sum_{i=1}^{n} x_{i}^{2} \sum_{i=1}^{n} y_{i}-\sum_{i=1}^{n} x_{i} \sum_{i=1}^{n} x_{i} y_{i}}{n \sum_{i=1}^{n} x_{i}^{2}-\sum_{i=1}^{n} x_{i} \sum_{i=1}^{n} x_{i}}
\end{gathered}
$$

Finally, the yaw angle is calculated with:

$$
\psi=\arctan (A)
$$

\section{IMPLEMENTATION}

The V4core (Fig. 1) mobile robot platform is used for implementation of the presented method and obtaining data in real-life scenarios. We used the planar segmentation algorithm from Point Cloud Library (PCL) [21] in the ground plane detection step of the method, and the V4R ${ }^{1}$-library's [22] camera tracker to obtain camera movements trajectory in two other steps.

\section{EXPERIMENT}

Three cameras are mounted at different heights and angles on the robot looking to the floor in front of it. Ground truth for $\mathrm{X}, \mathrm{Y}$ and $\mathrm{Z}$ lengths of these cameras are measured manually using tape measure and laser measuring tool and the ground truth angels are measured by cameras looking at a fiducial marker (similar to QR code) that is fixed in the environment of the robot. Afterwards this data is used to build a simulation model of the robot in GAZEBO simulation environment in which the proof-of-concept tests are conducted. Since the camera tracker is not able to work properly in simulation environment a piece of software is developed to calculate camera trajectory based on the tf tree of the robot and simulate the camera tracker. The outcome proved functionality of the method under ideal circumstances of simulation compare to ground truth data (cf. Fig. 7, 8, 9).

For real-life scenarios, two kinds of experiments are conducted in areas with mosaic and wooden floor structure (Fig. 6) several times for each of the three cameras. The

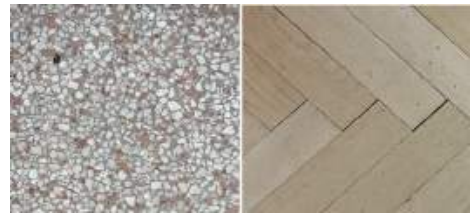

Fig. 6. Mosaic and wooden floor structure

results are gathered from the bottom camera at $0.44 \mathrm{~m}$, middle camera at $0.75 \mathrm{~m}$ and top camera at $1.33 \mathrm{~m}$ from the floor. The whole process of calibration for each camera took under three minutes. Fig. 7, 8, 9 demonstrate estimation error and standard error of it with respect to ground truth for each camera in both areas. Fig. 10, 11 illustrate comparison between the calculated ground truth camera trajectories and the obtained results from real camera tracker on both areas. These measurements are recorded during a circular path drive of the robot.

Bottom camera provides very good tracking results in both areas (cf. Fig. 10, 11). Because the camera tracker trajectories are almost in a perfect circle shape and fitted circles to them are very close to the ground truth trajectories. This type of good matching results in accurate estimation of $\mathrm{X}$ and $\mathrm{Y}$ lengths with less than $10 \mathrm{~mm}$ error (Fig. 7) in both areas. But the pose estimation error increased for the middle camera and

\footnotetext{
${ }^{1}$ Vision for Robotics group. ACIN, TU Vienna
} 

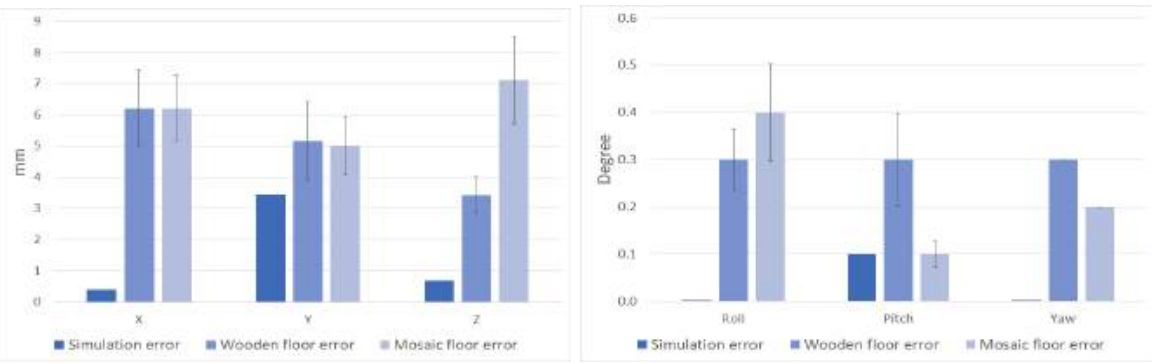

Fig. 7. Bottom camera pose error $\left(\mathrm{X}=0.28 \mathrm{~m}, \mathrm{Y}=-0.15 \mathrm{~m}, \mathrm{Z}=0.44 \mathrm{~m}\right.$, Roll $=1.1^{\circ}$, Pitch $=26.9^{\circ}$, Yaw $\left.=-29.8^{\circ}\right)$.
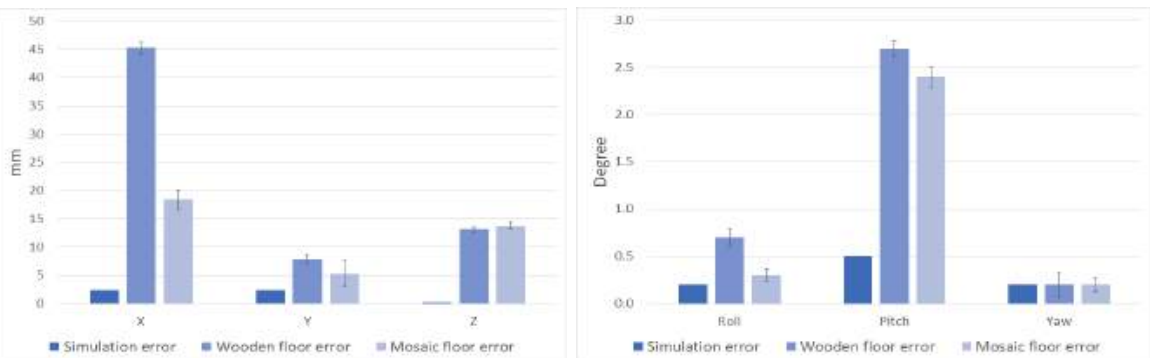

Fig. 8. Middle camera pose error $\left(X=0.115 \mathrm{~m}, \mathrm{Y}=0.02 \mathrm{~m}, \mathrm{Z}=0.755 \mathrm{~m}\right.$, Roll $=0.1^{\circ}$, Pitch $=34.4^{\circ}$, Yaw $\left.=0.1^{\circ}\right)$.
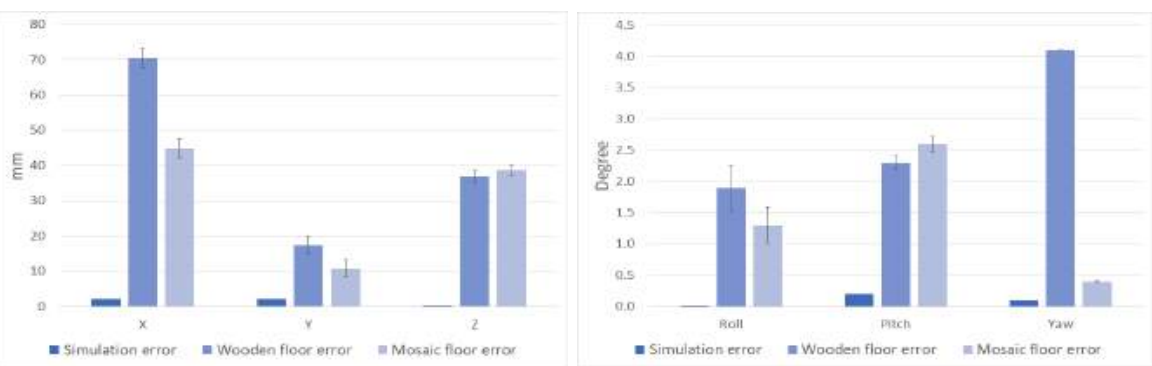

Fig. 9. Top camera pose error $\left(\mathrm{X}=0.173 \mathrm{~m}, \mathrm{Y}=0.02 \mathrm{~m}, \mathrm{Z}=1.333 \mathrm{~m}\right.$, Roll $=0^{\circ}$, Pitch $=51.6^{\circ}$, Yaw $\left.=0^{\circ}\right)$.

it increased dramatically for the top one (Fig. 8, 9), because the camera tracking error got bigger as the height of cameras from the floor increased (Fig. 10, 11). The better performance of the camera tracker on the mosaic floor area compared to the wooden one is also noticeable (cf. Fig. 10, 11) which leads to less estimation error for mosaic area. The reason behind this is that the mosaic floor has a lot more traceable features (texture) in its pattern than the wooden floor.

Another noticeable point in both scenarios is the increment of error in estimation of the $\mathrm{Z}$ length and the angels, when the camera is getting far from the floor. This result was expected because it is already shown in previous works when the distance between the 3D camera and the planar surface increases the depth accuracy of the sensor decreases [23].

\section{CONCLUSION}

This paper focused on presenting a novel autonomous and fast method for extrinsic calibration of a 3D camera on board of a mobile robot without any need for artificial targets, using camera motion estimation and robot's mobility. The simulation results proved the concept and the real-life scenarios also demonstrated that, with consideration of the accuracy range of the depth sensor and sufficient texture of the robot's working environment, it can provide good results in term of accuracy for practical cases. It is known from stereo systems that the floor always contains some texture or stains which are sufficient to be tracked contrary to walls, that might be really textureless. The method has a significant 

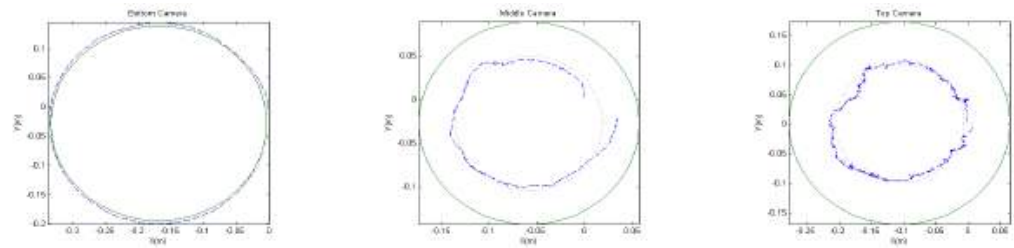

Fig. 10. Calculated ground truth (-), tracking trajectory (- - ) and circle fitting $(\cdots)$ result for three cameras mounted at different heights $(0.44 \mathrm{~m}$, 0.75 $\mathrm{m}, 1.33 \mathrm{~m}$ ) from wooden floor during a circular path drive of the robot.
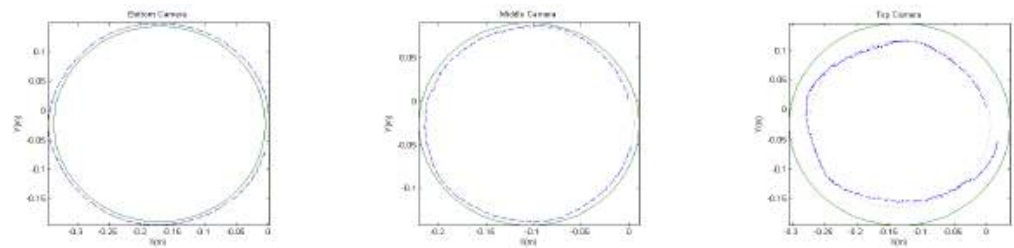

Fig. 11. Calculated round truth (-), tracking trajectory (- - ) and circle fitting ( $\cdots)$ result for three cameras mounted at different heights $(0.44 \mathrm{~m}, 0.75$ $\mathrm{m}, 1.33 \mathrm{~m}$ ) from mosaic floor during a circular path drive of the robot.

advance over present systems to use the existing environment itself as calibration pattern. Another advantage is its speed. The full calibration can be done under three minutes, unlike manual method which are much more slower. Furthermore, the robot can check its calibration at any time. Future works will be the studding effect of the intrinsic calibration of the $3 \mathrm{D}$ camera on the method and refinement of the method to live camera calibration during SLAM without need for any predefined paths.

\section{REFERENCES}

[1] F. Endres, J. Hess, J. Sturm, D. Cremers, and W. Burgard, "3-c mapping with an rgb-d camera," IEEE Transactions on Robotics, vol. 30 , no. 1 , pp. $177-187,2014$

[2] T. Fulhammer, R. Ambru, C. Burbridge, M. Zillich, J. Folkesson, N. Hawes, P. Jensfelt, and M. Vincze, "Autonomous learning of object models on a mobile robot," IEEE Robotics and Automation Letters, vol. 2, no. 1, pp. 26-33, 2017.

[3] K. Tateno, F. Tombari, and N. Navab, "Real-time and scalable incremental segmentation on dense slam," in 2015 IEEE/RSI International Conference on Intelligent Robots and Systems (IROS), 2015, pp. 44654472 .

[4] M. Nießner, M. Zollhöfer, S. Izadi, and M. Stamminger, "Real-time $3 \mathrm{~d}$ reconstruction at scale using voxel hashing," ACM Trans. Graph., vol. 32, no. 6, pp. 169:1-169:11, Nov. 2013.

[5] C. C. Wang, "Extrinsic calibration of a vision sensor mounted on a robot," IEEE Transactions on Robotics and Automation, vol. 8, no. 2, pp. 161-175, 1992.

[6] M. Pollefeys, R. Koch, and L. V. Gool, "Self-calibration and metric reconstruction in spite of varying and unknown internal camera parameters," in Sixth International Conference on Computer Vision (IEEE Cat. No.98CH36271), 1998, pp. 90-95.

[7] S. J. Maybank and O. D. Faugeras, "A theory of self-calibration of a moving camera," International Journal of Computer Vision, vol. 8 , no. 2, pp. 123-151, Aug 1992.

[8] Q.-T. Luong and O. Faugeras, "Self-calibration of a moving camera from point correspondences and fundamental matrices," International Journal of Computer Vision, vol. 22, no. 3, pp. 261-289, Mar 1997.

[9] S. N. Sinha, M. Pollefeys, and L. McMillan, "Camera network calibration from dynamic silhouettes," in Proceedings of the 2004 IEEE Computer Society Conference on Computer Vision and Pattern Recognition, 2004. CVPR 2004., vol. 1, 2004, pp. I-195-I-202 Vol.1.
[10] G. Carrera, A. Angeli, and A. J. Davison, "Slam-based automatic extrinsic calibration of a multi-camera rig," in 2011 IEEE International Conference on Robotics and Automation, 2011, pp. 2652-2659.

[11] S. Miller, A. Teichman, and S. Thrun, "Unsupervised extrinsic calibration of depth sensors in dynamic scenes," in 2013 IEEE/RSJ International Conference on Intelligent Robots and Systems, 2013, pp. $2695-2702$

[12] S. Li, P. N. Pathirana, and T. Caelli, "Multi-kinect skeleton fusion for physical rehabilitation monitoring," in 2014 36th Annual International Conference of the IEEE Engineering in Medicine and Biology Society, 2014, pp. 5060-5063.

[13] J. Levinson and S. Thrun, Unsupervised Calibration for Multi-beam Lasers. Berlin, Heidelberg: Springer Berlin Heidelberg, 2014, pp. 179-193.

[14] W. Maddern, A. Harrison, and P. Newman, "Lost in translation (and rotation): Rapid extrinsic calibration for 2d and 3d lidars," in 2012 IEEE International Conference on Robotics and Automation, 2012, pp. 3096-3102.

[15] G. Pandey, J. R. McBride, S. Savarese, and R. M. Eustice, "Automatic targetless extrinsic calibration of a $3 \mathrm{~d}$ lidar and camera by maximizing mutual information," in Proceedings of the Twenty-Sixth AAAI Conference on Artificial Intelligence, ser. AAAI'12. AAAI Press, 2012, pp. 2053-2059.

[16] J. Prankl, A. Aldoma, A. Svejda, and M. Vincze, "Rgb-d object modelling for object recognition and tracking," in 2015 IEEE/RSJ International Conference on Intelligent Robots and Systems (IROS), 2015, pp. 96-103.

[17] C. Tomasi and T. Kanade, "Detection and tracking of point features," International Journal of Computer Vision, Tech. Rep., 1991.

[18] E. Rosten, R. Porter, and T. Drummond, "Faster and better: A machine learning approach to corner detection," IEEE Transactions on Pattern Analysis and Machine Intelligence, vol. 32, no. 1, pp. 105-119, 2010.

[19] M. A. Fischler and R. C. Bolles, "Random sample consensus: A paradigm for model fitting with applications to image analysis and automated cartography," Commun. ACM, vol. 24, no. 6, pp. 381-395, June 1981.

[20] D. Eberly, "Least squares fitting of data," Chapel Hill, NC: Magic Software, 2000

[21] (2017) Point cloud library. [Online]. Available: http://pointclouds.org/about

[22] (2015) The vision4robotics library. [Online]. Available: https://www.acin.tuwien.ac.at/en/vision-for-robotics/softwaretools/v4r-library/ 
[23] H. Haggag, M. Hossny, D. Filippidis, D. Creighton, S. Nahavandi, and V. Puri, "Measuring depth accuracy in rgbd cameras," in 2013, 7th
International Conference on Signal Processing and Communication Systems (ICSPCS), 2013, pp. 1-7. 
